\title{
Evidence for Nonrandom Multiplicity of Gene Products in 22 Plant Genera
}

\author{
Charles F. Sing ${ }^{1}$ and George J. Brewer ${ }^{1}$ \\ Received 4 Sept. 1970-Final 10 Dec. 1970
}

Correlations in the number (multiplicity) of molecular forms among 10 enzymes have been estimated from a sample of plants representing 22 genera. Significant correlations in multiplicity among some of the 10 enzyme systems suggest a nonrandom organization of variation in number of molecular forms. An analysis of the correlations among enzymes of a glycolytic and Krebs cycle subset supports the occurrence of patterns of influence on multiplicity which correspond to the known metabolic relatedness of the enzymes. We interpret these data as evidence for organization of enzyme multiplicity. This property may be of adaptive value to the species.

\section{INTRODUCTION}

In recent years, electrophoretic studies have indicated that most proteins, including enzymes, occur in more than one molecular form in a species. Two broad categories of multiple forms of an enzyme (called isozymes) have been defined. First, allelic variation at a structural locus can produce variation in number of isozyme bands among individuals due to genetic segregation. For such loci, heterozygotes may show multiplicity in number of electrophoretically distinct molecular forms of an enzyme beyond that found in any homozygote. Recent studies have introduced the methodology for estimating the proportion of loci expected to be heterozygous in an individual of a given population (Harris, 1966; Lewontin and Hubby, 1966). Using gel electrophoresis of enzyme and protein variants, it has been estimated that in sexually reproducing populations $30-50 \%$ of the loci may be polymorphic (frequency of least frequent allele greater than 0.01 ) and that the average individual is heterozygous for

This research was supported by U.S.A.E.C. Contract AT(11-1)-1552, U.S.P.H.S. Grant AM 09381 and Rackham Faculty Award NSF-GU-3470-Project 131, and U.S.P.H.S. Career Development Award 1-K3-AM7959 (GJB).

1 Departments of Human Genetics and Medicine (Simpson Memorial Institute), University of Michigan Medical School, Ann Arbor, Michigan.

$\mathrm{C}$ 
$10-25 \%$ of these loci. Shaw (1969), Scandalios (1969), and Brewer and Sing (1969) have presented reviews of the available data from both plants and animals.

The second broad category of multiple forms includes those which do not vary among individuals of the population. The electrophoretically distinct nonsegregating multiple forms of lactic dehydrogenase ( $\mathrm{LDH})$ are an example. In contrast to the many studies of isozymes associated with allelic variation, little attention has been given to the evolutionary significance of this type of nonsegregating enzyme multiplicity. Nonsegregating isozymes may be the result of various states of polymerization of a single polypeptide subunit. They also may result from random (LDH) or nonrandom (hemoglobin) combinations of subunits which are structurally different. Gene duplication is the most likely mechanism for producing a number of slightly different polypeptide subunits in all individuals of the species. Brewer and Sing (1968) estimated that as many as $60 \%$ of the mammalian red blood cell enzymes show electrophoretically distinct multiple forms unrelated to heterozygosity. Later, Sing and Brewer (1969) found that $100 \%$ of the tissue enzymes of the predominantly inbreeding plant, wheat, showed such multiplicity. In most outbreeding species, there are many enzymes which have both segregating and nonsegregating forms (see Knutsen et al., 1969, for a discussion).

An important question is whether the multiplicity ${ }^{2}$ of molecular forms of an enzyme of a species is an evolutionary adaptation or meaningless biochemical redundancy. In this paper, we wish to evaluate the hypothesis that multiplicity for any one enzyme of a species might be selected as a coadaptation with other enzymes which have related functions because they are a part of an organized metabolic subsystem within the individuals of the species. Our null hypothesis is that the degree of multiplicity for each enzyme system is independent of that found at another. On the other hand, correlations in multiplicity between enzyme systems would be evidence to reject this hypothesis and suggest that systematic arrangement of protein multiplicity has occurred.

\section{MATERIALS AND METHODS}

\section{Species and Isozyme Techniques}

The 22 genera studied were chosen to represent a wide range of plant types (Table 1). A sample of $500 \mathrm{mg}$ of the entire organism was used in most cases. Exceptions were the woody plants, in which only leaves were taken, and unicellular species, where $500 \mathrm{mg}$ was not necessary to obtain an adequate sample of protein.

Electrophoretic techniques used here (phosphoglucomutase, PGM; hexokinase, HEX; glucose 6-phosphate dehydrogenase, G6PD; 6-phosphogluconate dehydrogenase, 6PGD; malic dehydrogenase, $\mathrm{MDH}$; alcohol dehydrogenase, $\mathrm{ADH}$; esterase, Est; alkaline phosphatase, Alk Ph; acid phosphatase, $\mathrm{A} \mathrm{Ph}$; leucine amino peptidase, LAP) are given by Brewer (1970). For this study, PGM, HEX, G6PD, 6PGD, and MDH were considered to be representative of the glycolytic-Krebs cycle (GK) set

${ }^{2}$ In this paper, we shall utilize the term multiplicity to mean the number of isozymes seen after an electrophoretic run. Hence, multiplicity in number of forms of a specific protein from both segregating and nonsegregating sources is considered as the measure of the biochemical diversity a species has available at the gene product level. 
Table I. The Average Number of Isozyme Bands for Each of 10 Enzyme Systems Measured on Representatives of 22 Plant Genera

\begin{tabular}{|c|c|c|c|c|c|c|c|c|c|c|}
\hline \multirow[b]{2}{*}{ Genus (common name) } & \multicolumn{10}{|c|}{ Enzymes } \\
\hline & PGM & HEX & $\begin{array}{l}\text { G6- } \\
\text { PD }\end{array}$ & $\begin{array}{l}\text { 6P- } \\
\text { GD }\end{array}$ & MDH & $\mathrm{ADH}$ & Est & $\begin{array}{c}\text { Alk } \\
\mathrm{Ph}\end{array}$ & $\mathrm{APh}$ & LAP \\
\hline Chara (green alga) & 4.0 & 5.2 & $0^{a}$ & 1.0 & 7.5 & 0 & 0 & 7.0 & 0 & 1.5 \\
\hline $\begin{array}{l}\text { Chlamydomonas (unicellular } \\
\text { alga) }\end{array}$ & 0 & 0 & 1.7 & 0.5 & 4.0 & 0 & 0 & 0 & 0 & 0 \\
\hline Cycas (cycad) & 6.5 & 6.5 & 5.5 & 1.5 & 7.0 & 0 & 4.0 & 1.5 & 0 & 3.5 \\
\hline $\begin{array}{l}\text { Degeneria (primitive angio- } \\
\text { sperm) }\end{array}$ & 0.6 & 0.8 & 0 & 0 & 2.2 & 0.2 & 1.3 & 3.5 & 0.2 & 3.0 \\
\hline Dryopteris (fern) & 0 & 0 & 0 & 0 & 0 & 0 & 0 & 3.5 & 0 & 2.0 \\
\hline Escherichia (bacterium) & 4.7 & 2.5 & 6.6 & 6.2 & 3.5 & 1.7 & 2.2 & 4.3 & 0 & 0.5 \\
\hline Franseria (composite) & 0 & 0.3 & 0 & 0 & 0.6 & 0.2 & 2.1 & 3.9 & 5.0 & 3.0 \\
\hline Funeria (moss) & 1.7 & 1.6 & 0 & 0.6 & 2.1 & 0 & 1.3 & 4.2 & 0 & 1.0 \\
\hline Lycopodium (clubmoss) & 2.0 & 1.0 & 3.2 & 3.5 & 5.3 & 2.2 & 1.6 & 3.6 & 1.0 & 0.5 \\
\hline Lygodium (primitive fern) & 0 & 0.2 & 0.1 & 0.2 & 1.3 & 0.3 & 4.7 & 4.7 & 1.0 & 4.5 \\
\hline Marchantia (liverwort) & 0.5 & 1.0 & 0 & 0 & 1.0 & 0 & 3.0 & 3.5 & 0 & 1.0 \\
\hline Neurospora (fungus) & 4.5 & 6.2 & 4.1 & 3.8 & 6.7 & 3.4 & 5.8 & 4.0 & 0 & 1.0 \\
\hline Oncidum (orchid) & 2.0 & 2.9 & 0 & 1.0 & 3.8 & 0.4 & 8.3 & 3.7 & 0 & 0 \\
\hline Pilularia (advanced fern) & 0 & 0 & 0 & 0.8 & 0 & 0.3 & 2.9 & 4.9 & 0 & 4.0 \\
\hline Podocarpus (conifer) & 1.8 & 1.9 & 0 & 0 & 0 & 0.5 & 2.0 & 2.0 & 0.3 & 2.5 \\
\hline Psilotum (whiskfern) & 8.7 & 10.0 & 2.0 & 3.0 & 10.5 & 0 & 4.5 & 2.0 & 0 & 2.0 \\
\hline Salmonella (bacterium) & 4.5 & 6.3 & 2.0 & 2.0 & 6.0 & 0 & 0 & 0 & 0 & 0 \\
\hline Selaginella (spikewort) & 2.5 & 2.0 & 1.0 & 0.5 & 5.5 & 0 & 1.0 & 4.0 & 2.0 & 1.0 \\
\hline Triticum (wheat) & 6.0 & 3.5 & 3.5 & 2.0 & 9.5 & 0 & 3.0 & 8.5 & 3.0 & 1.0 \\
\hline Urginea (lily) & 4.5 & 1.2 & 2.9 & 3.3 & 5.3 & 0 & 3.6 & 2.8 & 0.3 & 1.2 \\
\hline Zea $(\operatorname{corn})$ & 5.0 & 4.0 & 5.0 & 3.0 & 7.0 & 0 & 11.0 & 10.5 & 3.5 & 1.0 \\
\hline Zygnema (alga) & 1.7 & 0 & 0 & 0 & 1.0 & 0 & 0 & 0 & 0 & 1.0 \\
\hline
\end{tabular}

${ }^{a}$ Zeros indicate that no bands were detected.

of enzymes, whereas the others were included as a sample with miscellaneous functions and denoted the MISC subset.

The number of isozyme bands for each of three gel slots was counted for species 1-10 and for each of five slots for species 11-22 (one individual or sample for each slot). The average number of bands per slot is given in Table I for each species and is the variable of interest. Hence, the data consisted of a vector of 10 averages for each of the 22 genera.

\section{Data Analysis}

The object of the analysis of these data was to estimate the degree to which multiplicity in one enzyme system is related to the multiplicity in a second. Because the joint frequency of pairs of enzyme averages in the sample (Table I) did not approximate any known bivariate distribution, a nonparametric correlation analysis using Spearman's rank statistic, $r_{s}$, (Siegel, 1956) was employed. It was found that the product moment correlation matrix, based on the actual averages, was very similar to the 
matrix of $r_{s}$ coefficients based on ranking. However, because of the obvious nonnormality of the data, tests of significance of deviations from the null hypothesis of a zero correlation based on product moment correlation would yield invalid statements of probability. Partial rank correlation coefficients were derived using the standard relationship among total correlations (Morrison, 1967, p. 89). (See Patel, 1968, for a discussion about the use of this formulation when the measure of correlation is nonparametric.) Appendix Table P in Seigel (1956) was used to obtain the critical twotailed $r_{s}$ values.

Knowledge of the degree to which these correlations in average number of multiple forms derive from the segregating or nonsegregating source is not necessary for a test of our null hypothesis. It is clear that at present it is impossible to partition the total correlation into a portion due to allelic variation and a portion due to nonsegregating multiplicity because few genetic data are available for these systems for the majority of the species which comprise the sample studied here. However, it is reasonable to assume that most of the multiplicity is of the nonsegregating type because (1) about $70 \%$ of the systems examined in any one natural population have shown no electrophoretically detectable genetic variation and (2) current estimates indicate that on the average only $10-25 \%$ of the polymorphic enzyme loci are expected to be heterozygous in any one individual.

\section{RESULTS}

The total correlation matrix based on rankings of means is presented in Table II. All 10 correlations between pairs of glycolytic and Krebs cycle (GK) enzymes are significantly different from zero at the 0.02 level of probability. In contrast, none of the

Table II. The Total Rank Correlation Coefficients, $r_{s}$, for Five Glycolytic (and Krebs Cycle) and Five Miscellaneous Enzymes

\begin{tabular}{|c|c|c|c|c|c|c|c|c|c|c|}
\hline & \multicolumn{5}{|c|}{ Glycolytic and Krebs cycle } & \multicolumn{5}{|c|}{ Miscellaneous } \\
\hline & PGM & HEX & G6PD & 6PGD & $\mathrm{MDH}$ & $\mathrm{ADH}$ & Est & Alk $\mathrm{Ph}$ & $\mathrm{A} \mathrm{Ph}$ & LAP \\
\hline PGM & 1 & $0.89^{a}$ & $0.74^{a}$ & $0.75^{a}$ & $0.81^{a}$ & -0.08 & $0.39^{b}$ & 0.07 & 0.12 & -0.20 \\
\hline HEX & & 1 & $0.56^{a}$ & $0.63^{a}$ & $0.80^{a}$ & 0.02 & $0.39^{b}$ & 0.10 & 0.04 & -0.16 \\
\hline G6PG & & & 1 & $0.82^{a}$ & $0.68^{a}$ & 0.17 & $0.44^{b}$ & 0.14 & 0.28 & -0.19 \\
\hline 6PGD & & & & 1 & $0.69^{a}$ & 0.28 & $0.43^{b}$ & 0.26 & 0.04 & -0.34 \\
\hline $\mathrm{MDH}$ & & & & & 1 & -0.16 & 0.26 & 0.13 & 0.15 & -0.24 \\
\hline $\mathrm{ADH}$ & & & & & & 1 & $0.38^{b}$ & 0.29 & 0.28 & 0.16 \\
\hline Est & & & & & & & 1 & 0.35 & $0.38^{b}$ & 0.19 \\
\hline Alk $\mathrm{Ph}$ & & & & & & & & 1 & $0.43^{b}$ & 0.14 \\
\hline $\mathrm{APh}$ & & & & & & & & & 1 & 0.26 \\
\hline LAP & & & & & & & & & & 1 \\
\hline
\end{tabular}

${ }^{a}$ Significant at the 0.02 level of probability.

${ }^{b}$ Significant at the 0.10 level of probability. 
correlations between miscellaneous enzymes (MISC) were significant at the 0.02 level. Three of the latter were significant at the 0.10 level. The independence of the GK set and the MISC set is reflected by the small number, four of 25, of correlations between enzymes in the two sets which are significant at the 0.10 level of probability. No significant negative correlations were observed.

In Table III the partial rank correlations between glycolytic and Krebs cycle enzymes are presented. By inspection we note that PGM and HEX multiplicity is closely correlated in these 22 genera and that this total correlation is affected very little by fixing the variability in G6PD, 6PGD, or MDH. Likewise, of the other pairs, G6PD and 6PGD are most closely correlated, and this relationship is affected very little by fixing the variability in the other enzymes considered. The latter pair of enzymes are more closely correlated with PGM than HEX. The relationship between G6PD or 6PGD and HEX is drastically altered when the variability in PGM is removed, indicating that the correlation relationship may be affected by PGM. On the basis of this treatment of the data, a graphic representation of the closeness of relationship between PGM, HEX, G6PD, 6PGD, and MDH might be as shown in Fig. 1. We place MDH as shown because it is approximately equally correlated with PGM and HEX, and the partial correlations are equally altered by fixing the variability in the G6PD or 6PGD.

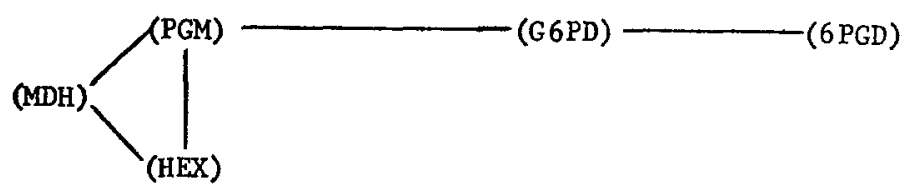

Fig. 1. Graphic representation of the relationship of PGM, HEX, G6PD, 6PGD, and MDH.

Table III. Total Rank Correlations and Partial Rank Correlations Between Five Glycolytic and Krebs Cycle Enzymes

\begin{tabular}{lcccccc}
\hline & \multicolumn{6}{c}{ Partial fixing } \\
\cline { 3 - 7 } & Total & PGM & HEX & G6PD & 6PGD & MDH \\
\hline PGM-HEX & 0.89 & - & - & 0.85 & 0.81 & 0.69 \\
PGM-G6PD & 0.74 & - & 0.64 & - & 0.33 & 0.44 \\
PGM-6PGD & 0.75 & - & 0.53 & 0.37 & - & 0.45 \\
PGM-MDH & 0.81 & - & 0.36 & 0.62 & 0.61 & - \\
HEX-G6PD & 0.56 & -0.32 & - & - & 0.10 & 0.04 \\
HEX-6PGD & 0.63 & -0.12 & - & 0.36 & - & 0.18 \\
HEX-MDH & 0.80 & 0.30 & - & 0.69 & 0.65 & - \\
G6PD-6PGD & 0.82 & 0.60 & 0.73 & - & $-\overline{-}$ & 0.66 \\
G6PD-MDH & 0.68 & 0.20 & 0.47 & - & 0.28 & - \\
6PGD-MDH & 0.69 & 0.21 & 0.40 & 0.32 & - & - \\
\hline
\end{tabular}




\section{DISCUSSION}

On the basis of the matrix of total correlations, we reject the general hypothesis that the joint distributions of the number of multiple molecular forms are independent. Specifically, total correlations indicate that the GK sample of enzymes are more closely correlated with one another than the sample of MISC enzymes.

By comparing the significant positive correlations within the GK set with correlations of the GK with the MISC enzymes, one has a measure of nonrandom organization attributable to the relatedness of the GK enzymes. The correlations between GK and MISC enzymes can be taken as a measure of the general latent correlation among all pairs of enzymes. Then, the increase in correlation among GK enzymes, above this baseline, can be considered that component of correlation which is attributable to the metabolic relationships which exist among GK enzymes. The average of all pairwise correlations among PGM, HEX, G6PD, 6PGD, and MDH is 0.74 , whereas the average correlation of these with MISC enzymes is 0.20 . The difference, 0.54 , estimates the increase in correlation among GK enzymes. We expect a difference this large less than $2 \%$ of the time by chance alone.

The meaning of the significant correlations among enzymes which can be classified as related is enhanced by the comparison with the concomitant absence of correlation among the so-called miscellaneous enzymes. We interpret the increase in correlation among related enzymes as evidence for selection of concomitant multiplicity in functionally related enzymes. In other words, the strong correlations in multiplicity between metabolically related enzymes suggest a set of enzymes (here the glycolytic and Krebs cycle) which may have evolved isozyme diversity as a part of an organized subsystem.

The biological relevance of enzyme multiplicity per se, whether the result of allelic variation or duplication of genes, is not at all clear. For reasons discussed above, the largest share of the multiplicity measured here is probably of the nonsegregating type. The genetic origin of nonsegregating enzyme multiplicity has been suggested as gene duplication (see Smithies, 1964, for a discussion). Studies intended to reveal the biological relevance of multiple forms arising in this manner have been limited to a few molecules. Hemoglobin is frequently cited as an example of the evolutionary significance of multiplicity arising from gene duplication. Here gene duplication has given rise to multiple forms of a protein (e.g., fetal and adult hemoglobin) which presumably have significant physiologic differences. Another example is LDH. Again, the origin of the LDH monomers as gene duplications has been confirmed in certain lower organisms (Ohno, 1970). Work with the LDH isozymes indicates that the amounts and activities of the various LDH isozymes differ among tissues in the body. It has been suggested that these differences are related to varying metabolic characteristics of the specific tissues.

Another approach for studying possible roles of nonsegregating multiplicity has been to characterize isozymes from inbreeding species, where genetic segregation is nonexistent or at least minimal. A study of the inbreeding wheat species by Sing and Brewer (1969) suggested that on the average there were more nonsegregating isozymes for each of 10 enzymes in leaf tissues of the broadly adapted Triticum species than in leaves of their more narrowly adapted Aegilops relatives. In general, the wheat 
species had a larger average number of isozymes and a greater proportion of enzymes showing multiplicities than outbreeding animal species.

A smaller portion of the multiplicity measured here is probably the result of heterozygosity for segregating alleles. Studies of the biological significance of polymorphic allelic variation have been of great interest. A short review of the contribution of these studies to understanding protein multiplicity follows. Theoretical studies by Kimura and Crow (1964) and Kimura (1968) followed by a review of the available data on proteins by King and Jukes (1969) have suggested the argument that much of the observed allelic variation responsible for polymorphic enzyme frequencies may not be selected upon by the environment. Instead, they argue these variations are probably functionally equivalent and are observations in the population of intermediate allele frequencies produced as a consequence of the process of genetic drift operating on recurrent neutral mutations (see Kimura and Ohta, 1969). This point of view implies that alternate forms of an enzyme do not represent symbols of information that "signal" different biological outcomes but are a measure of the entropy (noise) present in the living system. The necessity for "non-Darwinian" evolution at most of the observed polymorphic loci, it is argued, derives from theory that predicts that the average fitness for the population would be near zero if each polymorphic allele were being maintained by selection. However, data on the distribution of isozymes in nature have been interpreted by others as evidence for rejection of the hypothesis that genetic variations are functionally equivalent. Prakash et al. (1969) and O'Brien and MacIntyre (1969), measuring 24 and 10 enzyme loci, respectively, reported that the observed differences in gene frequencies among populations of $D$. pseudoobscura, D. melanogaster, and $S$. simulans are in direct opposition to the predictions of Kimura based on selective neutrality.

The manner of selection which could lead to such high levels of polymorphism is obscure. On one hand, it is argued that the overdominance and "hard" selection (Wallace, 1970), as might be characterized by a balanced lethal, can operate at only a fraction of the loci. Consequently, other mechanisms (see Dempster, 1955, for a review) based on systems of interaction among loci in time and space must be relevant. (Also see Sved et al., 1967, and the discussion of "soft selection" by Wallace, 1970.)

The analysis presented here utilizes a multivariate method of estimating the extent to which organization of biochemical diversity occurs among enzyme systems within individuals. To date, the usual approach has been to study the distributions of alternate forms of each protein considered separately. Nonrandom distributions of alternate forms of an enzyme among species, ecological niches, individuals, or sites within individuals is considered evidence for the operation of selection. Such analyses disregard the joint frequencies of alternate forms for pairs of enzymes which may indicate nonrandom internal organization. Each enzyme can be viewed as an element of a subsystem of the individual, the subsystem in our study being a metabolic pathway. If two enzymes operate as a part of the same subsystem and a correlation in multiplicity obtains, it suggests organization of multiplicity. Such interrelationships imply the operation of selection for coadaptation of enzymes at the biochemical level.

The selective mechanism by which variations are maintained for each enzyme may not be in terms of the independent contribution of each isozyme to the total phenotype. 
It seems more likely that selection among external phenotypes, measured as differential reproduction, may be translatable to selection on each enzyme only in terms of the contribution of the output of the subsystem of which the enzyme is a part to the reproductive performance of the individual. This implies that the isozyme may be an adaptation which contributes to the efficiency of the subsystem, and subsystems which vary in isozyme variability contribute differentially to the reproductive performance on which evolution is based.

How does the organization of enzyme multiplicity contribute to better-adapted subsystems? It would seem to us that evolution at the internal sublevel likely involves selection for reliable subsystems; the glycolytic pathway is an important example. The reliability of a subsystem can be measured in terms of the amount of information represented by the elements of which it is composed, i.e., multiple strategies for achieving a given function. Those subsystems with more alternative strategies to accomplish a successful output carry more information than those with few choices at each step in the process leading to the output. The availability of isozymes at each of the steps could lead to a successful operation of the glycolytic subsystem, for example, under a broader range of environments, including internal niches at the organ and cellular level. If we assume that the environments subject the component enzymes of a subsystem to roughly the same selection pressure, correlated multiplicity would be expected. As the environment of the subsystem changes, an alternate combination of isozymes could be utilized. Translated to probabilities, the subsystem with the greater number of combinations would have the higher probability of successfully achieving the necessary output for a given array of environments presented to the subsystem. From this, one would predict that subsystems which are subject to different degrees of environmental variation will differ from one another in the amount of multiplicity present for component enzymes.

Conjecture is widespread that much of the observed enzyme multiplicity may be evolutionary noise. One difficulty is that proof of this hypothesis is virtually impossible. The best approach is one which attempts to reject such a "null" hypothesis. Evidence for rejection for at least some enzymes is presented in this paper as correlations. We recognize that our approach has not differentiated segregating and nonsegregating multiplicities and does not address itself to the role of either source. However, more intensive research into the contributions of enzyme multiplicity per se to the organization of a successful living system is likely to provide the key to understanding the relevance of protein multiplicity.

\section{ACKNOWLEDGMENTS}

We are appreciative of the dedicated technical assistance of Sasha Ainsworth, David R. Bowbeer, and Lucia Brewer. We wish to thank the staff of the University of Michigan Botanical Garden for their assistance in growing the plants used in this study.

\section{REFERENCES}

Brewer, G. J. (1970). An Introduction to Isozyme Techniques, Academic Press, New York, 175 pp. Brewer, G. J., and Sing, C. F. (1968). The study of evolution through isozymes. J. Clin. Invest. 46: 11a. 
Brewer, G. J., and Sing, C. F. (1969). Survey of isozymes of human erythrocytes. In Yunis, J. J. (ed.), Biochemical Methods in Red Cell Genetics, Academic Press, New York, pp. 377-390.

Dempster, E. R. (1955). Maintenance of genetic heterogeneity. Cold Spring Harbor Symp. Quant. Biol. 20: 25 .

Harris, H. (1966). Enzyme polymorphisms in man. Proc. Roy. Soc. London Series B 164: 298.

Kimura, M. (1968). Genetic variability maintained in a finite population due to mutational production of neutral and nearly neutral isoalleles. Genet. Res. Cambridge 11: 247.

Kimura, M., and Crow, J. F. (1964). The number of alleles that can be maintained in a finite population. Genetics 49: 725 .

Kimura, M., and Ohta, T. (1969). The average number of generations until fixation of a mutant gene in a finite population. Genetics 61: 763 .

King, J. L., and Jukes, T. H. (1969). Non-Darwinian evolution. Science 164: 788.

Knutsen, C., Sing, C. F., and Brewer, G. J. (1969). Hexokinase isozyme variability in Drosophila robusta. Biochem. Genet. 3: 475.

Lewontin, R. C., and Hubby, J. L. (1966). A molecular approach to the study of genic heterozygosity in natural populations. II. Amount of variation and degree of heterozygosity in natural populations of Drosophila pseudoobscura. Genetics 54: 595.

Morrison, D. (1967). Multivariate Statistical Methods, McGraw-Hill, New York, 338 pp.

O'Brien, S. J., and MacIntyre, R. J. (1969). An analysis of gene-enzyme variability in natural populations of D. melanogaster and D. simulans. Am. Naturalist 103: 97.

Ohno, S. (1970). Evolution by Gene Duplication, Springer-Verlag, New York. 280 pp.

Patel, K. M. (1968). A nonparametric test for the multiple correlation. Ann. Math. Stat. 39: 690.

Prakash, S., Lewontin, R. C., and Hubby, J. L. (1969). A molecular approach to the study of genic heterozygosity in natural populations. IV. Patterns of genic variation in central, marginal, and isolated populations of Drosophila pseudoobscura. Genetics $61: 841$.

Scandalios, J. G. (1969). Genetic control of multiple molecular forms of enzymes in plants: A review. Biochem. Genet. 3: 37.

Shaw, C. R. (1969). Isozymes: Classification, frequency, and significance. Intern. Rev. Cytol. $25: 297$.

Siegel, S. (1956). Nonparametric Statistics for the Behavioral Sciences, McGraw-Hill, New York, $312 \mathrm{pp}$.

Sing, C. F., and Brewer, G. J. (1969). Isozymes of a polyploid series of wheat. Genetics 61: 391.

Smithies, O. (1964). Chromosomal rearrangements and protein structure. Cold Spring Harbor Symp. Quant. Biol. 29: 309.

Sved, J. A., Reed, T. E., and Bodmer, W. F. (1967). The number of balanced polymorphisms that can be maintained in a natural population. Genetics 55: 469.

Wallace, B. (1970). Genetic Load: Its Biological and Conceptual Aspects, Prentice-Hall, Englewood Cliffs, N.J. 